Cereal Research Communications 43(2), pp. 307-317 (2015)

DOI: $10.1556 / C R C .2014 .0038$

First published online 4 February, 2015

\title{
Comparison of Structural Development and Biochemical Accumulation of Waxy and Non-waxy Wheat Caryopses
}

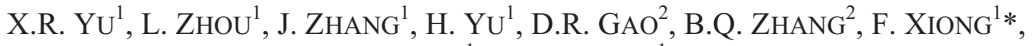 \\ Y.J. GU ${ }^{1}$ and Z. WANG ${ }^{1}$ \\ ${ }^{1}$ Key Laboratories of Crop Genetics and Physiology of the Jiangsu Province and Plant Functional Genomics \\ of the Ministry of Education, Yangzhou University, Yangzhou 225009, China \\ ${ }^{2}$ Key Laboratory of Wheat Biology and Genetic Improvement for Low \& Middle Yangtze Valley \\ (Ministry of Agriculture), Lixiahe Agricultural Institute of Jiangsu Province, No. 568, \\ Yangzijiang North Road, Yangzhou 225007, China \\ (Received 29 April 2014; Accepted 30 June 2014; \\ Communicated by J. Johnson)
}

\begin{abstract}
This study was conducted to compare structural development and biochemical accumulation of waxy and non-waxy wheat (NW) caryopses. The caryopses' microstructure of the waxy wheat (WW) and NW cultivars at different developmental stages were observed under light, fluorescence, and scanning electron microscope. The results were as follows: Compared with NW, WW had a shorter maturation duration, which was reflected in several following characteristics. Programmed cell death of the pericarp began earlier, and the chlorophyll-containing layer in the pericarp was smaller. Vacuoles in chalazal cells accumulated more tannins at different developmental stages. Starch granules and protein bodies in the endosperm showed a higher accumulation level in developing caryopses, and aleurone cells were larger in size with larger numbers of aleurone grains. An analysis of the element content indicated that the mineral elements $\mathrm{Mg}, \mathrm{P}, \mathrm{K}$, and $\mathrm{Ca}$ exhibited a higher content, while the heavy elements $\mathrm{Cr}, \mathrm{Cd}$, and $\mathrm{Pb}$ exhibited a lower content in the aleurone layer.
\end{abstract}

Keywords: waxy, non-waxy, wheat, microstructure, biochemical accumulation

Abbreviations: DAF, days after fertilization; EDS, energy dispersive X-ray spectroscope; NW, non-waxy wheat; FM, fluorescence microscope; LM, light microscope; SEM, scanning electron microscope; WW, waxy wheat

\section{Introduction}

Wheat (Triticum aestivum L.) is one of the oldest, most extensively cultivated, and nutritionally high valued crops in the world. Wheat from different regions has distinctive properties, which make it suitable for particular uses, such as bread or noodles (Zhao et al. 2013). Wheat is stable food in 43 countries, accounting for $35 \%$ of the total world population (Zhang et al. 2013). Commercial wheat cultivars are generally classified into four

* Corresponding author. E-mail: feixiong@yzu.edu.cn

0133-3720/\$20.00 C 2015 Akadémiai Kiadó, Budapest 
types: soft red winter, hard red winter, hard red spring, and durum wheat. In cereals, such as rice, barley, maize, and sorghum, many natural waxy mutants have been reported. These mutations, which lead to amylose-free varieties, are considered unlikely in wheat due to its hexaploid genome (Yoo and Jane 2002).

Starch, which accounts for approximately $63-72 \%$ of endosperm dry weight in mature caryopses, is the most abundant storage component, and is composed of two D-glucose homopolymers, amylose and amylopectin. Amylose is synthesized by granule-bound starch synthase (GBSS), also known as waxy protein. There are three genes encoding isoforms of GBSS. Common wheat, a hexaploid plant, has three waxy loci, $W x-A 1, W x-B 1$ and $W x-D 1$, located on homoeologous genomes, A, B and D, respectively (Yasui and Ashida 2011). When the three waxy loci are functional, the lines are considered to be wild-type. When one or two waxy loci are nonfunctional, they are called partially waxy. Wild-type and partially waxy wheat are collectively known as NW. Genotypes with null alleles at all three waxy loci, which results in amylose not being synthesized, are called WW (Hansen et al. 2010). Several nonfunctional and partially functional waxy alleles have been identified in bread wheat (Yasui 2006). Based on X-ray microtomography and scanning electron microscopy (SEM), Zhu et al. (2012) studied the kernel structure of high-amylose and wild-type (low-amylose) rice. High amylose rice had elongated and smaller starch granules with more air spaces inside the kernels resulting in an opaque grain, whereas wild-type rice had a tight endosperm with little air space and polygonal starch granules. One of two near-isogenic waxy wheat lines showed an increased arabinoxylan content in the wheat caryopses (Takata et al. 2007). Yasui et al. (1999) also demonstrated that in the kernel of waxy wheat mutant total starch content was lower and fat and $(1 \rightarrow 3),(1 \rightarrow 4)$-beta-D-glucan content were higher compared with its parent.

Previous studies focused on the qualities, properties and potential use of starch in bread and noodle production, and the grain protein content, flour yield, starch crystallinity, and starch pasting and thermal properties between WW and NW (Jonnala et al. 2010; Ma et al. 2013). However, no systematic comparison of the caryopsis microstructure and biochemical accumulation between WW and NW has been reported. To further understand the differences in functional and nutritional properties of the newly developed WW and NW, studying the ultrastructure of the caryopsis is imperative since it is associated with the quality and uses of different wheat. In this study, we investigated the differences in structural development and biochemical accumulation between WW and NW caryopses.

\section{Materials and Methods}

\section{Materials}

The plant materials used in this study, the WW cultivar Yang 05G68 with three null wx loci, $w x-A 1, w x-B 1$, and $w x-D 1$ and NW cultivar Yangmai 15 with three functional alleles at the $w x$ loci were courteously provided by Derong Gao, a research scientist from Lixiahe Regional Agricultural Research Institute of Jiangsu Province, China. They were grown from October 2012 to June 2013 in the experimental field of the Agricultural College, 
Yangzhou University, Yangzhou, China. To determine the date of anthesis, individual florets were marked with a pen and the plants were tagged.

\section{Histochemical observation of developing caryopses}

Fresh caryopses from different days after fertilization (DAF) were collected, cut breadthwise from the center, and stained by $\mathrm{I}_{2} / \mathrm{KI}$ solution $(0.3 \%$ iodine and $1 \%$ potassium iodide) at room temperature $\left(25^{\circ} \mathrm{C}\right)$ for $30 \mathrm{~s}$. The samples were photographed under a stereomicroscope (MZ6, Leica, Germany) equipped with a digital camera (Powershot S70, Canon, Japan).

\section{Fluorescence microscope (FM) observations of caryopsis}

Flesh caryopses were cut breadthwise by hand with a clean blade and 2 mm-thick slices were placed on glass slides. The samples were observed and photographed under FM (DMLB, Leica, Germany) equipped with a digital camera (PowerShot S50, Canon, Japan).

\section{Microstructural observations of caryopses}

Caryopses at different DAF were collected. A transverse slice $2 \mathrm{~mm}$ thick was cut from the center of each caryopsis and immediately soaked in a fixative containing $2.5 \%$ glutaraldehyde in phosphate buffer ( $\mathrm{pH} 7.2)$ at $4{ }^{\circ} \mathrm{C}$ for $4 \mathrm{~h}$. Then, samples were rinsed three times (10 min each) with $0.1 \mathrm{M}$ phosphate buffer ( $\mathrm{pH} 7.2)$ and dehydrated in an ethanol series of $20,40,60,80,90$ and $95 \%$ (10 min each) and 100\% (three times, 10 min each). The ethanol was then replaced by propylene oxide, and low glutinosity Spurr resin was used to infiltrate and embed materials. Finally, samples were polymerized at $70{ }^{\circ} \mathrm{C}$ for $12 \mathrm{~h}$ in an oven, then cut into 1 ìm slices using anultramicrotome (Ultracut R, Leica, Germany), stained with toluidine blue (1\%) for $5 \mathrm{~min}$, and photographed under a Leica DMLS light microscope (LM) equipped with a digital camera (E520, Olympus, Japan).

Ultrastructure observation and relative element content determination in the caryopses

Mature wheat grains were selected and fractured breadthwise in the center of the caryopses by hand using two pairs of pliers. The fractured caryopses, with the fractured surfaces upward, were stuck to the sample stage and sprinkled with gold at the fractured region. The structures of the caryopses were observed and photographed using SEM (XL-30 ESEM, Philips, Holland) equipped with a Charge Coupled Device (CCD) camera. The temperature of the frozen platform was $5{ }^{\circ} \mathrm{C}$ and the accelerating voltage was $20 \mathrm{kV}$. At the same time, the relative contents of mineral elements at different positions of the caryopses were determined using energy dispersive X-ray spectroscope (EDS) attached to the SEM. The relative contents were shown as atomic contents.

\section{Determination of amylose, amylopectin and protein content}

Mature wheat caryopses were harvested at 45 DAF. Wheat kernels were tempered to $15.5 \%$ moisture for $20 \mathrm{~h}$ and milled into flour in a mortar. Total protein content in wheat 
grain was calculated by the $\mathrm{N}$ concentration multiplied by the factor 5.7 (National Standard of the People's Republic of China, GB 5009.5-2010). N concentration was determined by using the Kjeldahl method (Yao et al. 2014). The protein content was expressed in $\%$ on a dry matter basis.

Amylose content, amylopectin content and total starch content were determined according to the method described by Zhu et al. (2007) with some modifications. Starch was first degreased by anhydrous ether and then $100 \mathrm{mg}$ of starch was added into $10 \mathrm{~mL}$ of $0.05 \mathrm{molL}^{-1} \mathrm{KOH}$ solution. Sample was diluted to $50 \mathrm{~mL}$ with distilled water and placed in water bath at $75{ }^{\circ} \mathrm{C}$ for $10 \mathrm{~min}$. A total of $2.5 \mathrm{~mL}$ of solution and $0.5 \mathrm{~mL} \mathrm{I}_{2}-\mathrm{KI}(0.2 \%$ iodine and $2 \%$ potassium iodide) were mixed and diluted to $50 \mathrm{~mL}$ with distilled water. At last, sample was used to determine amylose content and amylopectin content at wavelength $495-565 \mathrm{~nm}$ and $530-654 \mathrm{~nm}$, respectively. The recorded values were converted to percent of amylose by reference to a standard curve prepared with amylose from potato and amylopectin from corn (Sigma-Aldrich). Total starch content equals to amylose content plus amylopectin content.

\section{Statistical analyses}

The statistical analyses of the data were conducted using SPSS Statistics (version 19.0, International Business Machines Corporation, USA). The means were compared using Fisher's Protected Least Significant Difference (LSD) test at a 0.05 significance level. Origin 8.0 and Adobe Photoshop CS5 software packages were used to draw the figures.

\section{Results}

\section{Development of caryopses}

The morphological features of our two wheat cultivar caryopses, from fertilization to maturation, are shown in Fig. 1a. During caryopsis development, the caryopsis color changes from white to green, then from green to a yellowish green, and finally to yellow. At 12 $\mathrm{DAF}$, the caryopses color of WW turned from white to green while the caryopsis color of NW was still white. NW caryopses did not undergo the initial color change until 18 DAF. Nevertheless, no remarkable difference was observed in the size of the caryopses between WW and NW.

The wheat tissues that do not contain amylose stained red-brown by iodine. When amylose was present, the tissues stained blue-black. Figure $1 \mathrm{~b}$ shows the transverse sections of caryopses stained by $\mathrm{I}_{2} / \mathrm{KI}$. The area where starch accumulated included the pericarp and endosperm tissues. The pericarp in the caryopses of WW and NW both displayed the blue-black color from three DAF to 15 DAF. From 15 to 33 DAF, the endosperm tissues of WW stained red-brown while NW stained blue-black, and the colors gradually deepened as the caryopses developed. In plant organs, amylose is synthesized by a protein named granule-bound starch synthase (GBSS) which can be classified into two categories, GBSS I and GBSS II. The GBSS I gene is mainly expressed in storage organs, for example, seed endosperm of Gramineae crops and potato tubers, while the 
GBSS II gene is mainly expressed in non-storage tissues, for example leaves and pericarp. GBSS II was only detected in wheat pericarp but not in endosperm (Nakaruma et al. 1998). This may explain our results that WW only contained higher amylopectin content in the endosperm and no remarkably difference was found for amylose synthesis in the pericarp.

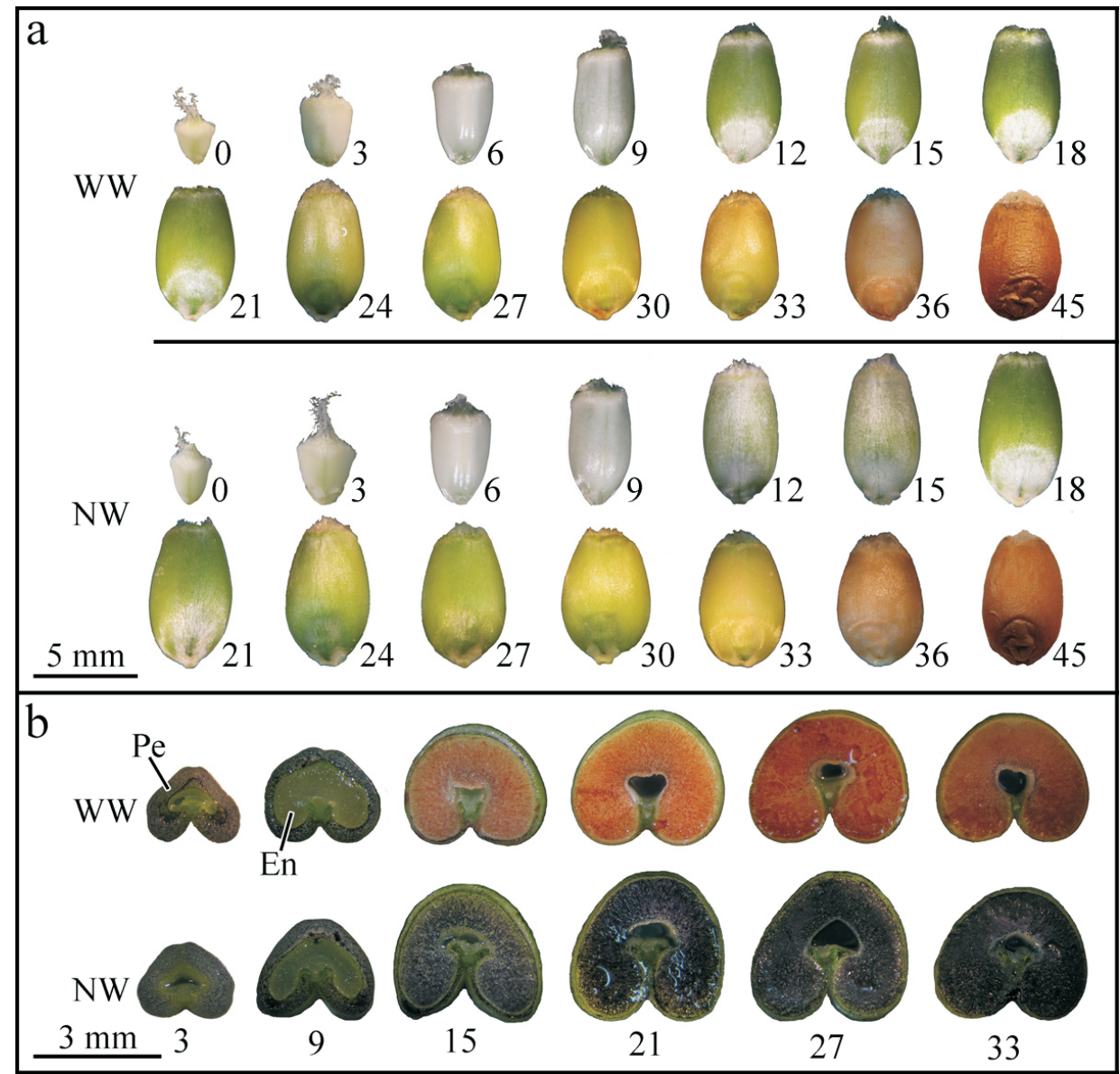

Figure 1. Wheat caryopsis growth. (a) Developing caryopsis profile. (b) Transverse sections of caryopses stained by $\mathrm{I}_{2} / \mathrm{KI}$ solution. The numbers below the images represent DAF of the caryopses. En, endosperm; Pe, pericarp

\section{Development of the pericarp}

The wheat pericarp, which lies outside the caryopsis, develops from the ovary wall and is considered to be composed of three parts: epicarp, mesocarp and endocarp. During wheat caryopsis development, the pericarp undergoes programmed cell death (Zhou et al. 2009), and different wheat cultivars display distinctive characteristics (Xiong et al. 2012). The 
physiological and anatomical functions of the pericarp are similar to those of leaves and also resemble those of storage organs (Müntz et al. 1978).

At five DAF, the pericarp of WW presented a stronger fluorescence signal and then began to degenerate. The space that formed by degeneration of the mesocarp (Sp) was clearly observed in WW, whereas no Sp could be found in the pericarp of NW (Figs 2a and 2e).

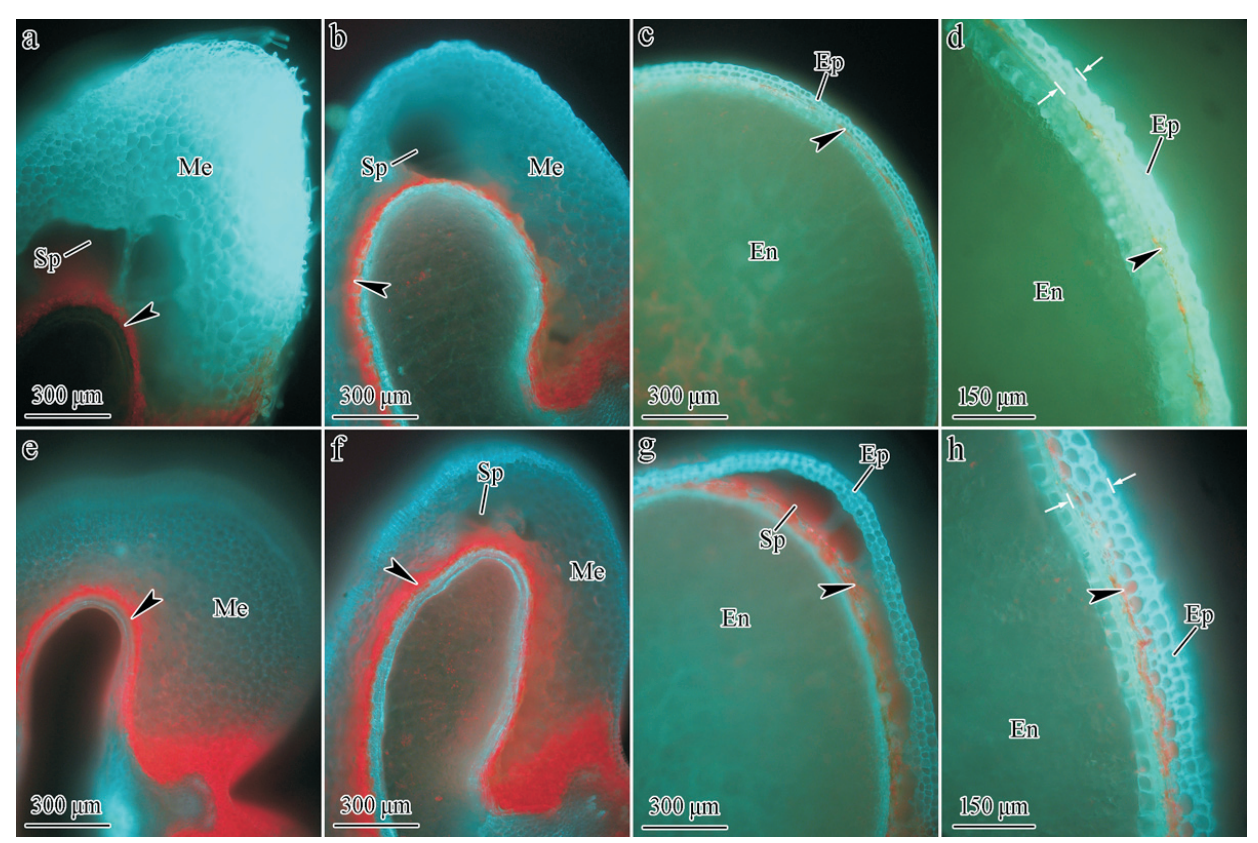

Figure 2. Fluorescence microscope images of the pericarp. (a) WW, 5 DAF; (b) WW, 8 DAF;

(c) WW, 16 DAF; (d) WW, 22 DAF; (e) NW, 5 DAF; (f) NW, 8 DAF; (g) NW, 16DAF; and

(h) NW, 22 DAF. En, endosperm; Ep, Epicarp; Me, mesocarp; and Sp, space formed by degeneration of pericarp cells. Black arrows indicate chloroplasts

At eight DAF, the pericarp of WW largely degenerated and cells decreased in comparison with those at five DAF (Fig. 2b). At this time, the pericarp of NW began to degenerate and some small Sps were detected (Fig. 2f). From 16 to 22 DAF, the mesocarp cells of WW had almost completely degenerated, and one or two epicarp remained (Figs $2 \mathrm{c}$ and 2d). In the pericarp of NW, by comparison, Sp can also be observed at 16 DAF (Fig. 2g), and the epicarp was thicker than that of WW (Fig. 2h).

Endocarp, the chlorophyll-containing layer where photosynthesis occurs, emits a red fluorescence under FM, and it is composed of tube and cross cells (Xiong et al. 2012). At five and eight DAF, the red fluorescence region in the pericarp of NW was significantly larger than that in WW (Figs 2a, 2b, 2e and 2f). From 16 to $22 \mathrm{DAF}$, the red fluorescence in the pericarp of WW gradually disappeared (Figs 2c and 2d). Compared with WW, 
a more obvious red fluorescence region was found in the pericarp of NW (Figs $2 \mathrm{~g}$ and $2 \mathrm{~h}$ ). The results indicated that the programmed cell death of the pericarp of WW was more rapid and the chlorophyll-containing layer was smaller than in NW.

\section{Development of the chalaza}

The chalaza, which is located between the vascular bundle and nucellar projection, plays an important role in assimilate translocation during the development of wheat endosperm (Cochrane 1983). Assimilates are imported into the developing endosperm first via a phloem pathway and then via a post-phloem pathway (Wang and Fisher 1994), in which the assimilates pass through the vascular parenchyma and several other parenchymal layers, the chalaza, the nucellar projection, the endosperm cavity, and the aleurone layer (Wang et al. 1998). The release of tannins, which accumulates in vacuoles of the chalazal cells, occurs 10 to 12 DAF after the cessation of dry matter accumulation in the wheat caryopses (Cochrane 1983). Later in caryopsis development, the cell wall thickened and stained a pale blue-green in a toluidine blue O solution (Zee and O'Brien 1970). Assimilates transported into the endosperm are considered to follow apoplastic and symplastic pathways until the chalazal cell walls thicken with lignin and suberin. At later stages of caryopsis development, the symplastic pathway is reduced by the thickened cell walls, and it is finally eliminated by the release of tannins from the vacuoles (Cochrane 1983).

At 12 DAF, vacuoles in the chalazal cells of WW accumulated brown-staining tannins but no tannins were found in NW (Figs S1a and S1b*). At 20 DAF, tannins had largely accumulated in the vacuoles of WW, much more so than in NW (Figs S1c and S1d). At 26 DAF, in the chalazal cells of WW, the vacuoles were filled with tannins that stained dark brown with a high intensity (Fig. S1e). Compared with 20 DAF, the cell walls had thickened and stained pale blue-green in a toluidine blue $\mathrm{O}$ solution. In the chalazal cells of NW, the cell wall had also thickened, but only little tannin vacuoles were found (Fig. S1f). The result indicated that the total amount of tannin was much lower in NW. As tannin was the substance impeding assimilate transport from the vascular bundle to the endosperm cavities, so this phenomenon might make NW being able to absorb more assimilate transported from vascular bundle.

\section{Development of the endosperm and aleurone layer}

During double fertilization, the triploid product of the second fertilization develops into endospermin the higher plant (Chaudhury et al. 1998). In cereals, which are a major source of food for mankind, the endosperm stores carbohydrates and proteins during caryopsis development. Also, the storage products, starch and protein, which accumulate in starch granules and protein bodies, are considered to be important nutrient sources during seed germination. The aleurone layer, which develops from surface endosperm cells, is a nutrient transporter that is able to transport the nutrient from endosperm cavities to endosperm during wheat caryopsis development. In different wheat cultivars, aleurone layers exhibited different sizes and shapes (Xiong et al. 2013).

\footnotetext{
* Further details about the Electronic Supplementary Material (ESM) can be found at the end of the article.
} 
Figure S2 shows the development of the endosperm and aleurone layers in our two wheat cultivars. At $12 \mathrm{DAF}$, starch granules and protein bodies accumulated in the endosperm of these wheat cultivars. Compared with NW, the endosperm of WW accumulated a greater number of protein bodies (Figs S2a and S2b). No remarkable differences were found in aleurone layers (Figs S2c and S2d). At 20 DAF, compared with NW, the number of starch granules and protein bodies was enriched in the endosperm cells of WW, and the aleurone cells were larger in size (Figs S2e, S2f, S2g and S2h). At 26 DAF, the endosperm cells of WW were highly enriched for starch granules and protein bodies. The latter were pushed together by the starch granules, deforming the cell's appearance into irregular shapes (Figs S2i and S2j). The aleurone cells were full of aleurone grains and were larger in size (Fig. S2k). The aleurone cells, containing fewer aleurone grains, were smaller in size (Fig. S21). Phytin particles, which accumulate in aleurone grains, are composed primarily of two elements, $\mathrm{P}$ and $\mathrm{Mg}$, with only small amounts of $\mathrm{K}$ and $\mathrm{Ca}$. Fatty acids, amino acids and minerals accumulate in aleurone cells, and can provide either nutrient resources for embryo early germination and hinder the transport of nutrients (Xiong et al. 2013). Thus, the differential development of endosperm and aleurone layers indicated that WW had a higher degree of biochemical accumulation.

Ultrastructure, amylose content, amylopectin content, total starch content and protein content of mature caryopses

It is known to all that waxy gene deficiency reduced the amylose content (Hansen et al. 2010). As shown in Table 1, in comparison with NW, WW shows a lower amylose content and a higher amylopectin content and total starch content. In high amylose content rice, the caryopses appear to be largely translucent, whereas in low amylose content rice they are opaque and the kernels contain numerous air spaces (Zhu et al. 2012). Vitreous endosperm has been suggested to result from the lack of air spaces (Hoseney 1986). Figure S3 shows the endosperm ultrastructure of a mature caryopsis under SEM. In the central endosperm of WW, more protein bodies filled the air space between starch granules and formed a tightly packed endosperm (Figs S3a and S3c). This structural conclusion was consistent with the result as shown in Table 1, which suggested that the protein content was higher in WW. On the surface of some parts of the starch granules many hollows were found, which might be formed by extrusion forces between the starch granules and protein bodies (Figs S3a and S3c). In the central endosperm of NW, the cells exhibited a looser structure that appeared to have more air space, and the surface of the starch granules was

Table 1. Content of amylose, amylopectin, total starch and protein and 1000-grain weight

\begin{tabular}{lccccc}
\hline Cultivars & $\begin{array}{c}\text { Amylose } \\
\text { content }(\%)\end{array}$ & $\begin{array}{c}\text { Amylopectin } \\
\text { content }(\%)\end{array}$ & $\begin{array}{c}\text { Total starch } \\
\text { content }(\%)\end{array}$ & $\begin{array}{c}\text { Protein } \\
\text { content }(\%)\end{array}$ & $\begin{array}{c}\text { 1000-grain } \\
\text { weight }(\mathrm{g})\end{array}$ \\
\hline WW & $0.99^{\mathrm{a}}$ & $73.21^{\mathrm{a}}$ & $74.20^{\mathrm{a}}$ & $12.66^{\mathrm{a}}$ & $38.55^{\mathrm{a}}$ \\
NW & $16.45^{\mathrm{b}}$ & $61.24^{\mathrm{b}}$ & $77.69^{\mathrm{b}}$ & $9.78^{\mathrm{b}}$ & $45.02^{\mathrm{b}}$ \\
\hline
\end{tabular}

Each data in the table are obtained from five replicates. Values in the same column followed by different superscript letters are significantly different, as determined by the LSD test $(P<0.05)$.

Cereal Research Communications 43, 2015 
smooth (Figs S3b and S3d). In accordance with the developing caryopsis, the aleurone layer cells of WW were larger in size compared with NW (Figs S3e and S3f). The structural characteristics indicated that WW was a hard wheat, and NW was a soft wheat.

\section{Distribution of elements in the caryopses}

Although the endosperm and aleurone layer both developed from a fertilized polar nucleus, they reportedly differ in development and element composition (Li et al. 2007). The concentrations of $\mathrm{P}, \mathrm{K}$, and $\mathrm{Mg}$ minerals in the aleurone layer and scutellum's cytoplasm were much higher than in the endosperm (Joyce et al. 2005). Xiong et al. (2013) also demonstrate that there are higher concentrations of the elements $\mathrm{Na}, \mathrm{Mg}, \mathrm{Si}, \mathrm{P}$, and $\mathrm{K}$ in the aleurone cells than in the endosperm cells.

In wheat grains, the concentrations of the heavy elements $\mathrm{Cd}$ and $\mathrm{Pb}$ occur in the order: aleuronic layer $>$ hypocotyls $>$ radicle $>$ scutellum $>$ plumule $>$ cortex $>$ endosperm (Zhang et al. 2010). The concentrations of the elements C, O, Mg, P, K, Ca, Cr, Cd, and Pb were determined using SEM equipped with EDS. The distributions of nine elements in the endosperm and aleurone layer are listed in Table S1. No significant differences between $\mathrm{WW}$ and $\mathrm{NW}$ were found in the endosperm or aleurone layer for elements $\mathrm{C}$ and $\mathrm{O}$. The other elements, $\mathrm{Mg}, \mathrm{P}, \mathrm{K}, \mathrm{Ca}, \mathrm{Cr}, \mathrm{Cd}$, and $\mathrm{Pb}$, were mainly concentrated in the aleurone layer, and the relative content of the atoms was greater in the aleurone layer than in the endosperm. In the aleurone layer, WW exhibited a higher content of elements Mg, P, K, and $\mathrm{Ca}$ and a lower content of elements $\mathrm{Cr}, \mathrm{Cd}$, and $\mathrm{Pb}$. Thus, compared with $\mathrm{NW}, \mathrm{WW}$ is a high-level mineral and low-level heavy elements cultivar, and its products are beneficial to human health.

\section{Discussion}

In this study, we investigated the structural development and biochemical accumulation between NW and WW and the results showed that the differences were reflected not only amylose contents but also the caryopsis development, which included the programmed cell death of the pericarp, development of chalazal cells and endosperm cells, and biochemical accumulation, such as starch granules, protein bodies, tannins, aleurone grains, and minerals. We also found that amylose content and total starch content was lower and amylopectin content and protein content was higher in WW. It is because of this possibility: Concomitant with inhibition of amylose synthesis, the ATP synthesized in photosynthesis was transferred to the synthesis of amylopectin and protein. This results in higher content of amylopectin and protein in mature caryopsis. Compared with NW, WW exhibited a shorter filling duration. This significant variation was reflected in the microstructure in developing and mature caryopses. The nutrients from the pericarp degradation enabled it to survive prior to the maturity of the caryopses, and the degradation also provided the nutrients for the growth of endosperm (Müntz et al. 1978). This may lead to an earlier onset of programmed cell death in the pericarp. Meanwhile, starch granule and protein body accumulation in the endosperm was greater, which resulted in a tightly packed endosperm with little air space. A greater accumulation of tannins in the chalazal cells indicated 
that dry matter accumulation was rapid, and this resulted in a lower 1000-grain weight (Table 1). The aleurone layer, which is known as a nutrient transporter, exhibited a larger size, where the mineral elements $\mathrm{Mg}, \mathrm{P}, \mathrm{K}$, and $\mathrm{Ca}$, and the heavy elements $\mathrm{Cr}, \mathrm{Cd}$, and $\mathrm{Pb}$ showed higher and lower concentrations, respectively.

\section{Acknowledgements}

This study was supported by the National Natural Science Foundation (Nos 31171482, 31270228, 31071341), Jiangsu Natural Science Foundation (BK2011445), Priority Academic Program Development from Jiangsu Government, China.

\section{References}

Chaudhury, A.M., Craig, S., Dennis, E.S., Peacock, W.J. 1998. Ovule and embryo development, apomixis and fertilization. Curr. Opin. Plant Biol. 1:26-31.

Cochrane, M.P. 1983. Morphology of the crease region in relation to assimilate uptake and water loss during caryopsis development in barley and wheat. Aust. J. Plant Physiol. 10:473-491.

Hansen, L.E., Jackson, D.S., Wehling, R.L., Graybosch, R.A. 2010. Functionality of chemically modified wild-type, partial waxy and waxy starches from tetraploid wheats. J. Cereal Sci. 51:409-414.

Hoseney, R.C. 1986. Principles of Cereal Science and Technology, First ed. American Association of Cereal Chemists. St. Paul, MN, USA.

Jonnala, R.S., MacRitchie, F., Smail, V.W., Seabourn, B.W., Tilley, M., La Fiandra, D., Urbano, M. 2010. Protein and quality characterization of complete and partial near-isogenic lines of waxy wheat. Cereal Chem. 87:538-545.

Joyce, C., Deneau, A., Peterson, K., Ockenden, I., Raboy, V., Lott, J.N.A. 2005. The concentrations and distributions of phytic acid phosphorus and other mineral nutrients in wild-type and low phytic acid Js-12-LPA wheat (Triticum aestivum) grain parts. Can. J. Bot. 83:1599-1607.

Li, C.Y., Feng, C.N., Wang, Y.L., Zhang, R., Guo, W.S., Zhu, X.K., Peng, Y.X. 2007. Differences of mineral element compositions and their contents among different positions of wheat grains. Plant Physiol. Commun. 43:1077-1081.

Ma, H.B., Zhang, X., Wang, C.G., Gao, D.R., Zhang, B.Q., Lv, G.F., Wu, R.L., Cheng, X.M., Wang, X., Cheng, S.H., Bie, T.D. 2013. Effect of $w x$ genes on amylose content, physicochemical properties of wheat starch, and the suitability of waxy genotype for producing Chinese crisp sticks. J. Cereal Sci. 58:140-147.

Müntz, K., Rudolph, A., Schlesier, G., Silhengst, P. 1978. The function of the pericarp in fruits of crop legumes. Die Kulturpflanz. 26:37-67.

Nakamura, T., Vrinten, P., Hayakawa, K., Ikeda, J. 1998. Characterization of a granule-bound starch synthase isoform found in the pericarp of wheat. Plant Physiol. 118:451-459.

Takata, K., Yanaka, M., Fujita, Y., Ishikawa, N. 2007. Evaluation of the grain and flour quality in near-isogenic wheat lines with waxy and double-null $w x$ proteins. Breeding Sci. 57:79-83.

Wang, N., Fisher, B.D. 1994. The use of fluorescent tracers to characterize the post-phloem transport pathway in maternal tissues of developing wheat grains. Plant Physiol. 104:17-27.

Wang, Z., Gu, Y.J., Li, W.F., Chen, G., Shi, H.Y., Chen, X.H. 1998. Development of wheat endosperm and pathway of nutrient entering the endosperm. Acta Agron. Sin. 24:536-545.

Xiong, F., Yu, X.R., Zhou, L., Wang, F., Xiong, A.S. 2012. Structural and physiological characterization during wheat pericarp development. Plant Cell Rep. 32:1309-1320.

Xiong, F., Yu, X.R., Zhou, L., Wang, Z., Wang, F., Xiong, A.S. 2013. Structural development of aleurone and its function in common wheat. Mol. Biol. Rep. 40:6785-6792.

Yao, X.Q., Chu, J.Z., He, X.L., Si, C. 2014. Grain yield, starch, protein, and nutritional element concentrations of winter wheat exposed to enhanced UV-B during different growth stages. J. Cereal Sci. 60:31-36.

Yasui, T. 2006. Waxy and low-amylose mutants of bread wheat (Triticum aestivum L.) and their starch, flour and grain properties. JARQ-Jpn. Agr. Res. Q. 40:327-331.

Cereal Research Communications 43, 2015 
Yasui, T., Ashida, K. 2011. Waxy endosperm accompanies increased fat and saccharide contents in bread wheat (Triticum aestivum L.) grain. J. Cereal Sci. 53:104-111.

Yasui, T., Sasaki, T., Matsuki, J. 1999. Milling and flour pasting properties of waxy endosperm mutant lines of bread wheat. J. Sci. Food Agric. 79:687-692.

Yoo, S.H., Jane, J.L. 2002. Structural and physical characteristics of waxy and other wheat starches. Carbohydr. Polym. 49:297-305.

Zee, S-Y., O'Brien, T.P. 1970. Studies on the ontogeny of the pigment strand in the caryopsis of wheat. Aust. J. Biol. Sci. 23:1153-1171.

Zhang, H.X., Zhang, W., Xu, C.Z., Zhou, X. 2013. Morphological features and physicochemical properties of waxy wheat starch. Int. J. Biol. Macromol. 62:304-309.

Zhang, J., Du, K., Chen, G., Wang, Y.P., Sun, G.R., Zhou, W.D. 2010. Distribution of Pb in Different Parts of Wheat Grains and Its Relationship with Other eight Elements. Bioinformatics and Biomedical Engineering (iCBBE), 2010, $4^{\text {th }}$ International Conference on. IEEE, Chengdu, China. pp. 1-4.

Zhao, H.Y., Guo, B.L., Wei, Y.M., Zhang, B. 2013. Multi-element composition of wheat grain and provenance soil and their potentialities as fingerprints of geographical origin. J. Cereal Sci. 57:391-397.

Zhou, Z.Q., Wang, L.K., Li, J.W., Song, X.F., Yang, C.N. 2009. Study on programmed cell death and dynamic changes of starch accumulation in pericarp cells of Triticum aestivum L. Protoplasma 236:49-58.

Zhu, L.J., Dogan, H., Gajula, H., Gu, M.H., Liu, Q.Q., Shi, Y.C. 2012. Study of kernel structure of high-amylose and wild-type rice by X-ray microtomography and SEM. J. Cereal Sci. 55:1-5.

Zhu, T., Jackson, D.S., Wehling, R.L., Geera, B. 2007. Comparison of amylose determination methods and the development of a dual wavelength iodine binding technique. Cereal Chem.85:51-58.

\section{Electronic Supplementary Material (ESM)}

Electronic Supplementary Material (ESM) associated with this article can be found at the website of CRC at http://www.akademiai.com/content/120427/

Electronic Supplementary Table S1. Distribution of mineral and heavy elements in the endosperm and aleurone layers

Electronic Supplementary Figure S1. Structure of chalazal cells. (a) WW, 12 DAF; (b) NW, 12 DAF;

(c) WW, 20 DAF; (d) NW, 20 DAF; (e) WW, 26 DAF; and (f) NW, 26 DAF. CH, chalazal; and TV, tannin vacuole. Red arrows marked in e and $\mathrm{f}$ indicate the thickened chalazal cell walls resulting from lignin and suberin deposition. The samples are stained by toluidine blue $\mathrm{O}$ solution

Electronic Supplementary Figure S2. Structure of the aleurone layer and endosperm. (a, c) WW, 12 DAF; (b, d) NW, 12 DAF; (e, g) WW, 20 DAF; (f, h) NW, 20 DAF; (i, k) WW, 26 DAF; and (j, l) NW, 26 DAF. $\mathrm{AG}$, aleurone grain; $\mathrm{AL}$, aleurone layer; $\mathrm{PB}$, protein body; and $\mathrm{SG}$, starch granule.

The samples are stained by toluidine blue $\mathrm{O}$ solution

Electronic Supplementary Figure S3. Scanning electron microscope images of the ultrastructure of mature wheat caryopses. (a, c) WW, central endosperm; (b, d) NW, central endosperm; (e) WW, aleurone layer; and (f) NW, aleurone layer. Red arrows indicate in $(a, c)$ hollows in the surface of starch granules,

and in $(b, d)$ the smooth surface of starch granules. Red asterisks indicate in $(b, d)$ the air space between starch granules and protein bodies. AL, aleurone layer; En, endosperm; PB, protein body; and SG, starch granule 